\title{
Importancia de la atención ortogeriátrica en pacientes con fractura de cadera. A propósito de un caso.
}

Importance of orthogeriatric care in patients with hip fracture. About a case.

Valeria Isabel Espín López. ${ }^{1}$, Karina Marisol Martínez Ortíz. ${ }^{2}$, Jessica Tatiana Reinoso Rivera. ${ }^{3}$ \& Esther Orfelina Sánchez Naranjo. ${ }^{4}$

\begin{abstract}
.
Hip fracture is a serious social health problem, the prevalence increases with age and susceptibility to the female sex. It increases the risk of mortality, dependency and institutionalization in older adults. The case of an 80-year-old woman who comes to the emergency room of a second-level hospital with a left intertrochanteric fracture, associated with controlled cardiac comorbidity, good family relationship, without alteration in the cognitive sphere, independent for basic and instrumental activities is
\end{abstract}

\section{Resumen.}

La fractura de cadera es un problema sociosanitario grave, la prevalencia aumenta con la edad y susceptibilidad al sexo femenino. Incrementa el riesgo de mortalidad, dependencia institucionalización en adultos mayores. Se reporta el caso de una mujer de 80 años que acude a emergencia de un hospital de segundo nivel con una fractura intertrocantérica izquierda, asociada a comorbilidad cardíaca controlada, buena relación familiar, sin alteración en la esfera cognitiva, independiente para las

\footnotetext{
${ }^{1}$ Universidad Técnica de Ambato, Facultad Ciencias de la Salud. Maestría en Atención Primaria de Salud mención en Gerontología. Ambato, Ecuador. espnvaleria@gmail.com

${ }^{2}$ Universidad Técnica de Ambato, Facultad Ciencias de la Salud. Maestría en Atención Primaria de Salud mención en Gerontología. Ambato, Ecuador. karynamartinez011285@ hotmail.com

${ }^{3}$ Universidad Técnica de Ambato, Facultad Ciencias de la Salud. Maestría en Atención Primaria de Salud mención en Gerontología. Ambato, Ecuador. tatty_1992@ hotmail.es

${ }^{4}$ Universidad Técnica de Ambato, Facultad Ciencias de la Salud. Maestría en Atención Primaria de Salud mención en Gerontología. Ambato, Ecuador. esther.sanchez@heg.gob.ec
} 
reported. of daily life. Left hip arthroplasty was performed, without pre and post-surgical evaluation by Geriatrics, or physiotherapy intervention, she was discharged from hospital 48 hours after surgery, without a home intervention plan. Patient died at 6 months at home, with functional deterioration, pressure ulcers and bedridden. The importance of orthogeriatrics as a model of care with an interdisciplinary approach aims to reduce hospital stay, avoid pre and postoperative complications, recover previous functionality with treatments in relation to the need of the elderly, achieving autonomy and social reintegration.

Keywords: elderly, fragility, hip fracture, rehabilitation, orthogeriatric unit. actividades básicas e instrumentales de la vida diaria. Se realiza artroplastia de cadera izquierda, sin valoración pre y postquirúrgica por Geriatría, ni intervención de fisioterapia, es dada de alta hospitalaria a las 48 horas luego de la cirugía, sin plan de intervención domiciliaria. Paciente fallece a los 6 meses en el domicilio, con deterioro funcional, úlceras por presión y encamamiento. La importancia de la ortogeriatría como modelo de atención con enfoque interdisciplinario tiene como finalidad reducir la estancia hospitalaria, evitar complicaciones pre y postoperatorias, recuperar la funcionalidad previa con tratamientos en relación con la necesidad del adulto mayor logrando autonomía y reinserción social.

Palabras claves: adulto mayor, fractura de cadera, fragilidad, rehabilitación, unidad de ortogeriatría.

\section{Introducción.}

El envejecimiento incluye varios cambios que inducen el riesgo de presentar patologías o la muerte. A nivel mundial la población afronta un proceso acelerado de incremento de la esperanza de vida. En Ecuador, la población de personas de 65 años es de 940.905, con una expectativa de vida de 75 años y para el año 2050 se prevé de 80.5 años (INEC, 2010). De acuerdo con esto hay una alta prevalencia de enfermedades, siendo una de ellas la fractura de cadera. Esta patología es un problema sociosanitario grave, frecuente en adultos mayores por la alta mortalidad que esta ocasiona. La incidencia de la fractura de cadera es mayor en mujeres que en hombres a partir de los 80 años (Gallardo \& Clavel, 2020).

Dado estos avances en la expectativa de vida reflejados de igual forma en la población de nuestro país en vías de desarrollo, es menester proporcionar la atención integral de salud en base al perfil epidemiológico de éste grupo etareo donde se recalca: el número de 
adultos mayores con osteoporosis, caídas que trae como consecuencia las fracturas de cadera (Marco, Galán Olleros, Mora Fernández, \& Mora-Fernández, 2019).

Esta enfermedad es un acontecimiento desafortunado con un impacto evidente en la calidad de vida e independencia de los adultos mayores y sus familias (Bachiller Caño, Soler de la Paz, Jiménez Méndez, \& Díaz Hernández, 2020).

Dentro de las consecuencias postfractura se encuentran los reingresos, alteraciones en la composición corporal (mineral óseo, muscular y grasa), caídas, úlceras de presión, infecciones, sobrecarga al cuidador, reintervenciones, así como el incremento en el uso de servicios de salud. Por lo tanto, es necesario identificar, diseñar, establecer y evaluar la eficacia de intervenciones con el objetivo de optimizar su recuperación post-fractura (de Miguel Artal et al., 2018).

Debido a la complejidad del paciente geriátrico hospitalizado se instaura las unidades de ortogeriatría con la finalidad de preparar al paciente para la cirugía, evitar las complicaciones médicas antes y después de la intervención, acortar la tasa de defunciones asociada a la fractura, restablecer la funcionalidad y disminuir días de hospitalización (Tarazona-Santabalbina, Belenguer-Varea, Rovira, \& Cuesta-Peredó, 2016). A pesar del auge de la acción interdisciplinaria en el ámbito sanitario, existe poca evidencia del efecto de las unidades ortogeriátricas en Latinoamérica (Viveros-García, 2019).

\section{Metodologia.}

Estudio descriptivo retrospectivo, presentación de un caso clínico de fractura de cadera y la importancia de la ortogeriatría.

\section{Resultados.}

Paciente femenina de 80 años, ecuatoriana, nacida y residente en la parroquia Atahualpa del cantón Ambato - Ecuador, instrucción primaria incompleta, católica, lateralidad diestra, ocupación anterior floricultura, dependiente económicamente de sus hijos, vive con su esposo y su hija en una propiedad arrendada en el tercer piso. Con antecedentes patológicos personales de hipertensión arterial, desde hace 20 años, controlada con Losartán 50mg al día; sin antecedentes patológicos quirúrgicos.

\section{Hábitos}

- Defecatorio: una vez al día

- Miccional: de 3 a 5 veces al día

- Nocivos: Exposición a carburantes de biomasa por 40 años aproximadamente.

Situación geriátrica basal: sueño irregular, acompañada de despertares de 3 a 4 veces, sin alteración en la esfera cognitiva, independiente para las actividades básicas e instrumentales de la vida diaria. 
Motivo de consulta: dolor en cadera y miembro inferior izquierdo.

Enfermedad Actual: Paciente hace aproximadamente 9 horas antes de su ingreso, sufre caída de su propia altura en el baño, permanece 6 horas sobre superficie, es trasladada por familiar al servicio de Emergencia de un Hospital de segundo nivel de atención, por presentar dolor intenso según escala de EVA 9/10, impotencia funcional de miembro inferior izquierdo y edema local. Se realizó radiografía de pelvis y miembro inferior izquierdo. Es ingresada al servicio de Traumatología con diagnóstico de fractura intertrocanterica izquierdaTronzo III B.

Examen físico: signos vitales: PA: 146/98 mmHg, FC: 88 lpm, FR: 22 rpm, temperatura: $36^{\circ} \mathrm{C}$, saturación de oxígeno: $88 \%, \mathrm{FiO}_{2:} 21 \%$.

Paciente álgica, lúcida, orientada en las tres esferas, hidratada, hipotérmica.

Acortamiento de miembro inferior izquierdo, rotación externa, impotencia funcional, equímosis, edema local, limitación a movimientos activos y pasivos. Resto de la exploración dentro de la normalidad.

Examenes complementarios: leucocitos: $5.200 \mathrm{~K} / \mathrm{ul}$, hemoglobina: $12 \mathrm{mg} / \mathrm{dl}$, hematocrito: 38\%, TPP: 28 segundos, TP: 12 segundos, urea: $27,4 \mathrm{mg} / \mathrm{dl}$, creatinina: $0,80 \mathrm{mg} / \mathrm{dl}$, glucosa: $99,7 \mathrm{mg} / \mathrm{dl}$.

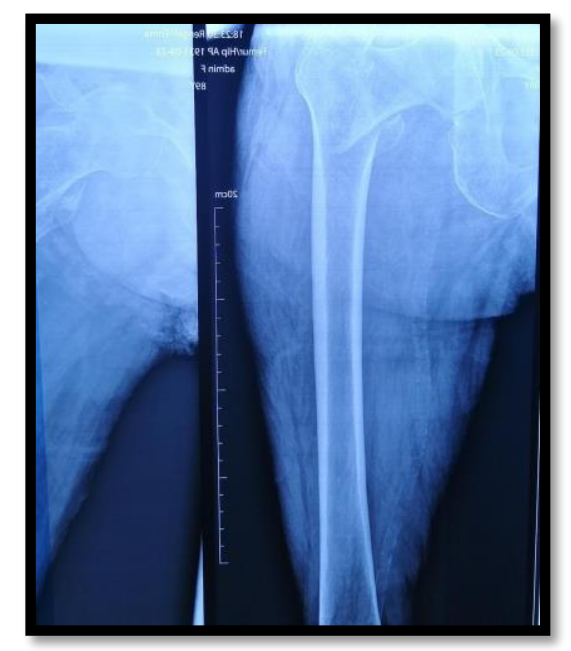

Figura 1. Fractura intertrocanterica izquierda Tronzo III B.

Fuente: Hospital público de segundo nivel de atención Ambato (2020).

El servicio de Traumatología decide intervención quirúrgica emergente. Se realiza artrosplatía de cadera izquierda sin complicaciones. Durante su estancia no se realizó una valoración Geriátrica integral ni terapia física con el fin de iniciar un plan de rehabilitación inmediato. A las 48 horas posteriores a la cirugía es dada de alta, con medicación analgésica. 
En el primer nivel de atención el personal de salud realizó una visita domiciliaria a los 7 días posteriores al egreso hospitalario, donde indicaron analgesia y actividad física. Paciente fallece a los 6 meses con cuadro de deterioro funcional, úlceras por presión y encamamiento de tres semanas.

\section{Discusión.}

Las caídas están relacionadas con traumatismos en adultos mayores que ingresan a los servicios de emergencia, se considera que se producen cada 13 segundos y cada 20 minutos fallece una persona (Ayala Guilcapi, Tituaña Vega, \& Tituaña Vega, 2020; Calero, López Cala, Ortega R, \& Cruz Lendínez, 2016).

Este síndrome geriátrico conlleva a fracturas de cadera, en un $75 \%$ al $95 \%$ por caídas desde su propia altura. La mortalidad en adultos mayores con fractura es de $31 \%$ en el primer mes, del 50\% a los 6 meses y el 56\% al año (Forster \& Calthorpe, 2000). Esta situación es relevante al aumentar el riesgo de muerte entre 3 a 4 veces más que para aquellos adultos mayores sin fractura de cadera (Ayala Guilcapi et al., 2020; Dinamarca Montecinos, Améstica Lazcano, \& Carrasco Buvinic, 2018; Henderson et al., 2017).

Las fracturas de cadera en adultos mayores frágiles traen consigo consecuencias físicas, psicológicas y sociales, aumentando el costo sociosanitario (Bielza et al., 2018; SáezLópez et al., 2019). Además de los gastos indirectos que se generan para las familias que deben atender a un adulto mayor con un grado de dependencia grave (Duarte-Flores, 2018).

La tasa de complicaciones luego de una fractura de cadera es del 45-57\%, dentro de las cuales están las infecciones del tracto respiratorio en un $13 \%$, la demencia con el $24 \%$, caídas recurrentes en el 12\% y úlceras de decúbito con el 33\% (Rapp et al., 2020). El $10,9 \%$ de los adultos mayores pueden sufrir una nueva fractura posterior al primer año si no recibe cuidados especiales a largo plazo (Venegas-Sanabria, Lozano-Rengifo, CepedaAlonso, \& Chavarro-Carvajal, 2018).

La ortogeriatría tiene como objetivo la pronta reinserción del paciente a la sociedad, manteniendo al máximo la capacidad funcional y evitar complicaciones (Abrahamsen, Nørgaard, Draborg, \& Nielsen, 2019). Sin embargo, sólo el 50\% de los pacientes que se recuperan alcanzan una condición física adecuada, un nivel de deambulación e independencia para las actividades de la vida diaria (Baroni et al., 2019). La combinación de un servicio de ortogeriatria con la atención primaria ayuda a aplacar problemas de comunicación, al poner a los profesionales de salud en contacto directo con la atención continua y monitoreo de los adultos mayores (Duaso et al., 2018).

El modelo ortogeriátrico reduce en 38 minutos la estancia en el servicio de emergencia y reduce la mortalidad a un año posterior a la cirugía (Schuijt et al., 2020). También, coordina la resolución quirúrgica en las primeras 24-48 horas después del ingreso, una valoración geriátrica integral, elaboración de un plan de rehabilitación individual, el 
apoyo brindado por el terapeuta ocupacional, una planificación del alta, seguimiento por atención primaria de salud, con opción de rehabilitación física y atención domiciliaria (Pioli, Bendini, Pignedoli, Giusti, \& Marsh, 2018) .

El personal de enfermería que forma parte del equipo de ortogeriatría debe liderar una intervención educativa con la finalidad de aumentar el autocuidado del paciente, siempre que la situación basal así lo permitiera, durante el proceso de hospitalización (pre y postquirúrgico) y aún más en la fase de alta (Viveros-García, 2019). El seguimiento debe estar enfocado no solo en la persona, sino también se debe valorar el entorno que rodea al paciente, la funcionalidad, el apoyo que recibe y la ayuda con la que debe contar para su reinserción en la sociedad (Fernández-Ibáñez et al., 2017).

El abordaje fisioterapéutico en ortogeriatría debe ser inmediato al post-operatorio (mismo día de la intervención), centrado en las movilizaciones adecuadas, tratamiento del dolor, cambios posturales, énfasis en sedestación e inicio precoz de la marcha con ayudas técnicas (andador o muletas), así como la formación al paciente y a la familia son de vital importancia para que el usuario sea dado de alta previo a una valoración fisiátrica y continuar con el seguimiento ambulatorio (Werner et al., 2020; Yeap, Hew, \& Chan, 2017).

El caso presentado permitió detectar falencias en la atención integral, las mismas que repercutieron en la rehabilitación funcional del paciente. Sin embargo, pese a que se contaba con el servicio de Geriatría no se interconsultó para una evaluación del estado general de la adulta mayor. En este contexto, al exitir un equipo de ortogeriatría el paciente hubiera tenido mejor pronótico.

La escasa comunicación entre el equipo interdisciplinario hizo que se omita la interconsulta al servico de fisiatría perdiendo este recurso tan imprescindible como es la terapia física, cuyo objetivo primordial es mantener el nivel de fuerza muscular anterior a la fractura. Asimismo, la atención recibida en el primer nivel fue deficiente de tal forma, que la paciente recibió una sola visita domiciliaria durante los 6 meses antes de su fallecimiento. Al no existir un seguimiento continuo, se paso por alto las complicaciones que agrabaron la condición de salud provocando dismovilidad y úlceras por presión con un desenlace fatal.

\section{Conclusiones.}

- La relevancia de la ortogeriatría reside en una comunicación asertiva entre el equipo interdisciplinario para un manejo global del paciente con fractura de cadera tanto a nivel hospitalario como el seguimiento en el primer nivel de atención.

- Al trabajar con un personal de salud debidamente capacitado en ortogeriatría se obtiene un buen pronóstico de vida del paciente, una recuperación inmediata y por ende disminuyen las complicaciones.

- El seguimiento de los pacientes en atención primaria de salud es un eje relevante 
para la recuperación biopsicosocial del paciente.

\section{Referencias bibliográficas.}

Abrahamsen, C., Nørgaard, B., Draborg, E., \& Nielsen, M. F. (2019). The impact of an orthogeriatric intervention in patients with fragility fractures: A cohort study. BMC Geriatrics, 19(1). https://doi.org/10.1186/s12877-019-1299-4

Ayala Guilcapi, I., Tituaña Vega, K. A., \& Tituaña Vega, A. P. (2020). Fractura de cadera y artroplastia en adulta mayor centenaria. Reporte de caso y revisión de literatura. ARS MEDICA Revista de Ciencias Médicas, 45(4). https://doi.org/10.11565/arsmed.v45i4.1736

Bachiller Caño, R., Soler de la Paz, Y., Jiménez Méndez, P. A., \& Díaz Hernández, D. (2020). Fractura de cadera en ancianos. European Journal of Health Research, 6(1). https://doi.org/10.30552/ejhr.v6i1.197

Baroni, M., Serra, R., Boccardi, V., Ercolani, S., Zengarini, E., Casucci, P., ... Ruggiero, C. (2019). The orthogeriatric comanagement improves clinical outcomes of hip fracture in older adults. Osteoporosis International, 30(4). https://doi.org/10.1007/s00198-019-04858-2

Bielza, R., Fuentes, P., Blanco Díaz, D., Moreno, R. V., Arias, E., Neira, M., ... Gómez Cerezo, J. F. (2018). Evaluación de las complicaciones clínicas de los pacientes con fractura de cadera y sus factores asociados en una Unidad de Agudos de Ortogeriatría. Revista Española de Geriatría y Gerontología, 53(3). https://doi.org/10.1016/j.regg.2018.01.002

Calero, J., López Cala, G., Ortega R, A., \& Cruz Lendínez, A. (2016). Prevención de caídas en el adulto mayor. European Journal of Investigation in Health, Psychology and Education, 6(2).

de Miguel Artal, M., Roca Chacón, O., Martínez-Alonso, M., Serrano Godoy, M., Mas Atance, J., \& García Gutiérrez, R. (2018). Hip fracture in the elderly patient: Prognostic factors for mortality and functional recovery at one year. Revista Espanola de Geriatria y Gerontologia, 53(5), 247-254. https://doi.org/10.1016/j.regg.2018.04.447

Dinamarca Montecinos, J., Améstica Lazcano, G., \& Carrasco Buvinic, A. (2018). Mortalidad intrahospitalaria en adultos mayores chilenos con fractura de cadera: incidencia, causas y otros elementos de interés. Revista Chilena de Ortopedia y Traumatología, 59(02). https://doi.org/10.1055/s-0038-1668593

Duarte-Flores, J. O. (2018). The orthogeriatric units in Mexico are a priority. Revista Medica Del Instituto Mexicano Del Seguro Social, 56(4). 
Duaso, E., Formiga, F., Marimón, P., Sandiumenge, M., Salgado, M. T., Murga, V., ... Sellarès, R. (2018). Advantages of care for patients with hip fractures in the acute geriatric unit: Hip study Anoia. Geriatrics and Gerontology International, 18(3). https://doi.org/10.1111/ggi.13191

Fernández-Ibáñez, J. M., Morales-Ballesteros, M. C., Crespo-Romero, E., GómezGómez, S., Fraga-Fuentes, M. D., Cruz-Tejado, J., ... García-Baltasar, M. M. (2017). Orthogeriatric activity in a general hospital of Castilla-La Mancha, Spain TT - Actividad ortogeriátrica en un hospital general de Castilla-La Mancha. Revista Espanola de Cirugia Ortopedica y Traumatologia, 61(2).

Forster, M. C., \& Calthorpe, D. (2000). Mortality following surgery for proximal femoral fractures in centenarians. Injury, 31(7). https://doi.org/10.1016/S00201383(00)00049-8

Gallardo, P., \& Clavel, O. (2020). FRACTURA DE CADERA Y GERIATRÍA, UNA UNIÓN NECESARIA. Revista Médica Clínica Las Condes, 31(1). https://doi.org/10.1016/j.rmclc.2019.09.004

Henderson, C. Y., Shanahan, E., Butler, A., Lenehan, B., O’Connor, M., Lyons, D., \& Ryan, J. P. (2017). Dedicated orthogeriatric service reduces hip fracture mortality. Irish Journal of Medical Science, 186(1). https://doi.org/10.1007/s11845-016-14533

INEC. (2010). Proyecciones Poblacionales |. Proyecciones Poblacionales.

Marco, F., Galán Olleros, M., Mora Fernández, J., \& Mora-Fernández, J. (2019). FRACTURA DE CADERA: EPIDEMIA SOCIO-SANITARIA DEL SIGLO XXI EN EL PRIMER MUNDO. ANALES RANM, 135(03), 203-210. Retrieved from https://analesranm.es/revista/2018/135_03/13503rev01

Pioli, G., Bendini, C., Pignedoli, P., Giusti, A., \& Marsh, D. (2018). Orthogeriatric comanagement - managing frailty as well as fragility. Injury, 49(8). https://doi.org/10.1016/j.injury.2018.04.014

Rapp, K., Becker, C., Todd, C., Rothenbacher, D., Schulz, C., König, H. H., ... Büchele, G. (2020). The association between orthogeriatric co-management and mortality following hip fracture:an observational study of 58000 patients from 828 hospitals. Deutsches Arzteblatt International, 117(4). https://doi.org/10.3238/arztebl.2020.0053

Sáez-López, P., Ojeda-Thies, C., Alarcón, T., Muñoz Pascual, A., Mora-Fernández, J., González de Villaumbrosia, C., ... González-Montalvo, J. I. (2019). [Spanish National Hip Fracture Registry (RNFC): First-year results and comparison with other registries and prospective multi-centric studies from Spain]. Revista Espanola de Salud Publica, 93. 
Schuijt, H. J., Kusen, J., van Hernen, J. J., van der Vet, P., Geraghty, O., Smeeing, D. P. J., \& van der Velde, D. (2020). Orthogeriatric Trauma Unit Improves Patient Outcomes in Geriatric Hip Fracture Patients. Geriatric Orthopaedic Surgery and Rehabilitation, 11. https://doi.org/10.1177/2151459320949476

Tarazona-Santabalbina, F. J., Belenguer-Varea, Á., Rovira, E., \& Cuesta-Peredó, D. (2016). Orthogeriatric care: Improving patient outcomes. Clinical Interventions in Aging, Vol. 11. https://doi.org/10.2147/CIA.S72436

Venegas-Sanabria, L. C., Lozano-Rengifo, M. J., Cepeda-Alonso, L., \& ChavarroCarvajal, D. A. (2018). Factores asociados a complicaciones intrahospitalarias en ancianos sometidos a cirugía por fractura de cadera. Revista Española de Geriatría y Gerontología, 53(3). https://doi.org/10.1016/j.regg.2017.06.004

Viveros-García, J. (2019). Ortogeriatría en México: un futuro posible. Med Int Méx. 2019 Enero-Febrero, 35(1). https://doi.org/10.24245/mim

Werner, M., Krause, O., Macke, C., Herold, L., Ranker, A., Krettek, C., \& Liodakis, E. (2020). Orthogeriatric co-management for proximal femoral fractures. Can two additions make a big difference? BMC Musculoskeletal Disorders, 21(1). https://doi.org/10.1186/s12891-020-03392-1

Yeap, S. S., Hew, F. L., \& Chan, S. P. (2017). Reply on "Combined orthogeriatric and fracture liaison service for improved postfracture patient care." Osteoporosis and Sarcopenia, 3(3). https://doi.org/10.1016/j.afos.2017.07.003

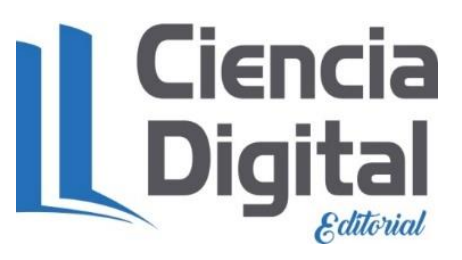




\section{PARA CITAR EL ARTÍCULO INDEXADO.}

Espín López, V. I., Martínez Ortíz, K. M., Reinoso Rivera, J. T., \& Sánchez Naranjo, E. O. (2021). Importancia de la atención ortogeriátrica en pacientes con fractura de cadera. A propósito de un caso. Anatomía Digital, 4(2), 132-141. https://doi.org/10.33262/anatomiadigital.v4i2.1689

\section{$\lfloor$ Ciencia}

El artículo que se publica es de exclusiva responsabilidad de los autores y no necesariamente reflejan el pensamiento de la Revista Anatomía Digital.

El artículo queda en propiedad de la revista y, por tanto, su publicación parcial y/o total en otro medio tiene que ser autorizado por el director de la Revista Anatomía Digital.
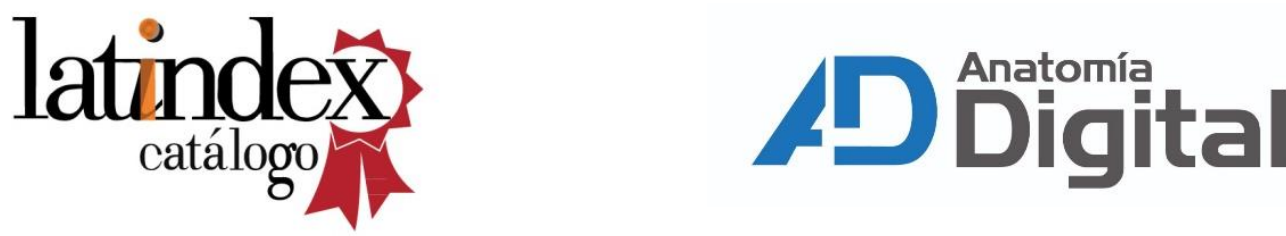